\title{
BAND-OFFSET AND ELECTRONIC PROPERTIES OF TYPE II PbTe/PbS SUPERLATTICES*
}

\author{
F.F. Sizov, J.V. Gumenjuk-Sichevskaya, V.V. Tetyorkin \\ AND V.V. ZABUDSKY
}

\begin{abstract}
Institute of Semiconductor Physics of the National Ukrainian Academy of Sciences 252028 , pr. Nauki 45, Kiev, Ukraine
\end{abstract}

\begin{abstract}
Semimetallic conductivity was observed in $\mathrm{PbTe} / \mathrm{PbS} \mathrm{SL}$ obtained by a "hot wall" technique on (001) $\mathrm{KCl}$ substrates with (001) PbTe buffer layers. From the analysis of $R_{\mathrm{H}}(H)$ and $\rho_{\perp}(H)$ dependencies by the Monte-Carlo fitting procedure the band offset $\Delta E_{\mathrm{v}}=0.32 \pm 0.05 \mathrm{eV}$ was obtained ( $T=$ $77 \mathrm{~K}$ ) and it was proved that these SLs are type II "misaligned" structures. The calculations of the band structure of $\mathrm{PbTe} / \mathrm{PbS}$ SLs showed that in such SLs a semimetal-semiconductor transition should occur for layer thicknesses of about $60 \div 70 \AA$.

PACS numbers: $68.65 .+\mathrm{g}$
\end{abstract}

Investigated up to now IV-VI superlattices (SLs) as a rule belong to type I SLs [1] except PbTe/SnTe system which forms type II "misaligned" SL [2]. Type II SLs offer some interests for infrared (IR) detector applications as it is possible to realize in such kind of systems favorable optical properties for long-wavelength detector applications through the valence to conduction band optical transitions between the states in alternate layers due to overlap of the envelope wave functions (see, e.g. [3]). The band gap in such structures occurs between the electron states localized in one types of layers and the hole states localized in remaining layers. $\mathrm{PbTe} / \mathrm{SnT}$ Te type II SLs however could not be used as effective photodetectors because of very high hole concentrations inherent to SnTe layers. It is supposed that type II IV-VI SLs also can be realized in $\mathrm{PbTe} / \mathrm{PbS}$ system, as these semiconductors differ much by the affinity energies $\left(\chi_{\mathrm{PbTe}}=4.6 \mathrm{eV}[4]\right.$, $\left.\chi_{\mathrm{PbS}}=3.9 \div 4.2 \mathrm{eV}[5]\right)$.

$\mathrm{PbTe}$ and $\mathrm{PbS}$ compounds differ much by the lattice constants $\left(a_{\mathrm{PbTe}}=\right.$ $6.460 \AA$ and $a_{\mathrm{PbS}}=5.94 \AA$ ) that should cause the arising of misfit dislocations at the interface. But as it was earlier shown for this system [6] the misfit dislocations are generated only in a thin layer near the interface. Beyond this thin highly deteriorated layer with "wood" of dislocations [6] the layers are unstrained and one can suppose to obtain the perfect layers with good electrical characteristics.

*This work was partly supported by the grant No. $2.2 / 11$ from the Ukrainian Committee of Science and Technology. 
$\mathrm{PbTe} / \mathrm{PbS}$ SLs with the number of periods up to $N=100$ were prepared by the "hot wall" epitaxy technique in a vacuum chamber with the gas pressure of $10^{-5} \div 10^{-6}$ torr at substrate temperatures not exceeding $T=350^{\circ} \mathrm{C}$. In this temperature range the metallurgical boundary between the $\mathrm{PbTe}$ and $\mathrm{PbS}$ layers should be very pronounced due to the absence of the intermixture between the constituents at temperatures $T<400^{\circ} \mathrm{C}$. The presence of the misfit dislocations at the interface in $\mathrm{PbTe} / \mathrm{PbS}$ system also prevail the intermixture of the components [6].

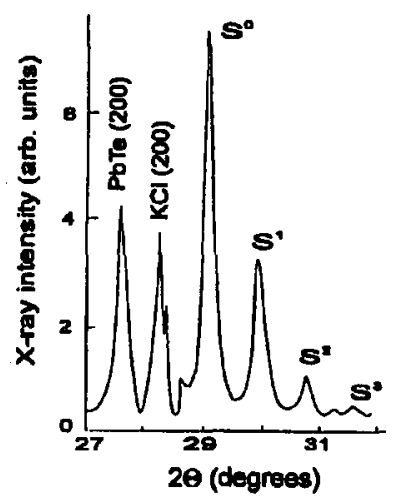

Fig. 1. Experimental X-ray spectra of 100 -period $\mathrm{PbTe} / \mathrm{PbS}(60 \AA / 60 \AA) \mathrm{SL}$ on $\mathrm{KCl}$ substrate with $d \approx 1 \mu \mathrm{m} \mathrm{PbTe}$ buffer layer.

The SLs grown exhibit up to four regularly spaced X-ray SL satellites (see Fig. 1). The appearance of numerous sharp, regularly spaced X-ray satellites is an indication of high structure quality SLs with little constituents interdiffusion [7]. Growth of SLs commenced with deposition of $\left(10^{3} \div 10^{4}\right) \AA$ buffer PbTe or PbS layers a top of the $(100) \mathrm{KCl}$ substrates. The thickness of $\mathrm{PbTe}$ or $\mathrm{PbS}$ layers in SLs varied between 60 and $500 \AA$. The total thickness of $\mathrm{PbTe} / \mathrm{PbS}$ SLs with buffer layers was in the range of $2 \div 3 \mu \mathrm{m}$.

Studies of the angle dependencies of transverse magnetoresistance showed a very pronounced epitaxy technique and confirmed quasi-two-dimensional character of conductivity in $\mathrm{PbTe} / \mathrm{PbS}$ SLs.

Up to now the band offset in $\mathrm{PbTe} / \mathrm{PbS}$ is not established. To obtain the quantitative values of band offset in these objects the studies of the temperature and magnetic field dependencies of Hall coefficient and transverse magnetoresistance were carried out.

To analyze the experimental dependencies in $\mathrm{PbTe} / \mathrm{PbS}$ SLs the multilayer conductivity model [8] was applied and the Monte-Carlo fitting procedure was used to find the equations solutions for this multilayer conductivity model.

The Monte-Carlo simulation method has been chosen to find the solution of the system of equations for transverse magneto-resistance and the Hall coefficient for multilayer structure [8] 


$$
\begin{aligned}
& \frac{\rho_{\perp}}{\rho_{0}}=\frac{\sum_{i} d_{i} N_{i} \mu_{i}(0)}{\sum_{i} d_{i} N_{i} \mu_{i}(H)}\left[1+\left(\mu_{\mathbf{H}} H\right)^{2}\right], \\
& R_{\mathrm{H}}=\rho_{\perp}(H) \mu_{\mathrm{H}},
\end{aligned}
$$

where the Hall mobility of a structure with different types of carriers in layers is determined by the expression $\mu_{\mathrm{H}}=\sum_{i} d_{i} N_{i} \mu_{i}^{2}(H) / \sum_{i} d_{i} N_{i} \mu_{i}(H)$. The values of carrier mobilities $\mu_{i}(H)$ and $\mu_{i}^{2}(H)$ in the magnetic field are defined by $\mu_{i}(H)=$ $\left\langle\mu_{i}(0) /\left(1+\mu_{i}^{2}(0) H^{2}\right)\right\rangle, \mu_{i}^{2}(H)=\left\langle\mu_{i}^{2}(0) /\left(1+\mu_{i}^{2}(0) H^{2}\right)\right\rangle$.

Experimentally determining the layer thicknesses and the resistivity, it is possible to obtain the basic electrical characteristics of each separate layer in the multi-layer structure, such as carrier concentrations of each SL layer $N_{i}$ and their mobilities $\mu_{i}$ from experimental $R_{\mathrm{H}}(H)$ and $\rho_{\perp}(H)$ dependencies (Eq. (1)) [8] by the Monte-Carlo fitting procedure.

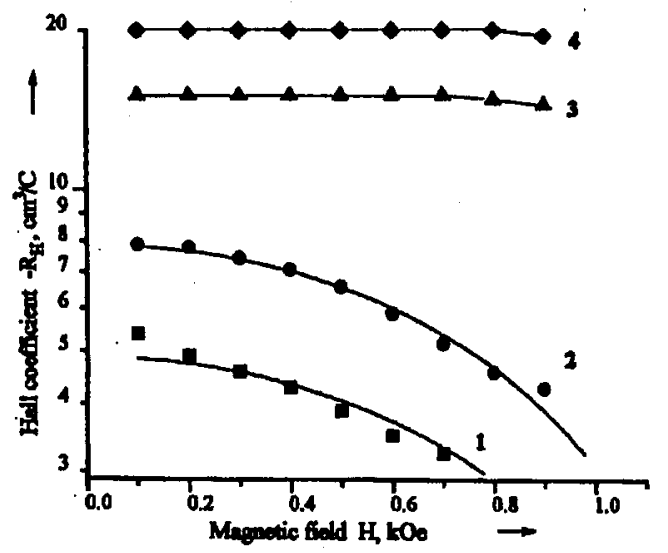

Fig. 2. Magnetic field dependencies of the Hall coefficient. $(1,2)-\mathrm{PbTe} / \mathrm{PbS} \mathrm{SL}$, (3) - PbS film, (4) - PbTe/PbS $(1.5 \mu \mathrm{m} / 1.5 \mu \mathrm{m})$ heterostructure.

The Hall coefficient and magnetoresistance dependencies on magnetic field were measured for $\mathrm{PbTe} / \mathrm{PbS} \mathrm{SLs}$, $\mathrm{PbS}$ film and one $\mathrm{PbS} / \mathrm{PbTe}$ heterostructure (see Fig. 2). There exists an essential difference in magnetic field dependencies for SLs, films and heterostructures. For last two objects there is almost no $R_{\mathbf{H}}(H)$, $\rho_{\perp}(H)$ dependencies which is characteristic for films and heterostructures in the case of in-plane transport. For SLs the $R_{\mathrm{H}}(H)$ and $\rho_{\perp}(H)$ dependencies are very strong even in weak magnetic fields which is characteristic for systems with two types of carriers (electrons and holes) with different mobilities (see e.g. [9]).

The Monte-Carlo simulation procedure can be fitted with the experimental data only in the case, when in $\mathrm{PbS}$ there are holes and in $\mathrm{PbTe}$ there are electrons. In these growth conditions used the separate $\mathrm{PbS}$ or $\mathrm{PbTe}$ layers were grown only of $n$-type conductivity. Thus, the change of the conductivity type in PbS layers, when they form a SL with PbTe layers, can be only in case when the electrons from $\mathrm{PbS}$ layers overflow to $\mathrm{PbTe}$ layers. It is possible only in case when these materials form a type II "misaligned" structure, and so the top of $\mathrm{PbS}$ valence 
band is above the bottom of the $\mathrm{PbTe}$ conduction band. Then, calculating the position of the Fermi level in the SL one can obtain the band-offset $\left(\Delta E_{\mathrm{v}}\right)$ in this system. The valence band offset obtained $\Delta E_{\mathrm{v}}=0.32 \pm 0.05 \mathrm{eV}$.

Using the values of the band-offset obtained, the band structure of $\mathrm{PbTe} / \mathrm{PbS}$ SLs was calculated. The band structure calculations of $\mathrm{PbTe} / \mathrm{PbS}$ SL with [100] growth direction were made in the envelope-function approximation [10] taking into account the nonparabolicity of the components bands near extremal $L$-points of the Brillouin zone in the framework of two-band Kane model.

We have calculated the band spectra of $\mathrm{PbTe} / \mathrm{PbS} \mathrm{SL}$ for different layer thicknesses and for temperatures $T=77$ and $300 \mathrm{~K}$. For layer thicknesses greater than $60 \AA$ the $\mathrm{PbTe} / \mathrm{PbS}$ SL without the buffer layer must exhibit a semimetallic conductivity due to the hybridization of $s$-type valence band states of $\mathrm{PbS}$ and $p$-type conductivity band states of $\mathrm{PbTe}$ at $T=77 \mathrm{~K}$. At higher temperatures the overlap decreases, and the transition to the state with the semimetallic conductivity must occur at higher layer thicknesses for the SL without the buffer layer. The presence of the buffer layer changes the situation, as for any $\mathrm{PbTe}$ layer thicknesses and for $\mathrm{PbS}$ thicknesses $\geq 35 \AA$ there would always be the $n$-type conductivity in $\mathrm{PbTe}$ buffer layer and the $p$-type conductivity in PbS SL layers. Thus such kind of a system will be a semimetallic one.

For the thicknesses $a \simeq b \leq 60 \AA$ the $\mathrm{PbTe} / \mathrm{PbS}$ SL itself must go over to the semiconductor one and can be used for the design of IR-photosensitive structures. Estimations of the interband absorption coefficient $\alpha$ have shown that $\alpha \simeq 10^{3} \mathrm{~cm}^{-3}$ at wavelength cutoffs of about $10 \mu \mathrm{m}$, which is a sufficient value for IR-photodetector applications.

The optical absorption spectra of $\mathrm{PbTe} / \mathrm{PbS}$ SLs investigated in this work did not reveal any SL absorption, as this to be observed, one need to have the SL with the period smaller than $120 \AA$ as to have the $\mathrm{PbS}$ valence band states lower than the PbTe conduction band states. But SLs with $d \leq 120 \AA$ were not obtained by the "hot wall" technique due to rather large layers growth rates $(\leq 8 \AA / \mathrm{sec})$ and inertia of the growth system used, which does not allow one to obtain the thin layers $(<60 \AA)$ with great accuracy needed for this case.

\section{References}

[1] F.F. Sizov, A. Rogalski, Progr. Quantum Electron. 17, 93 (1993).

[2] A. Ishida, M. Aoki, H. Fujiyasu, J. Appl. Phys. 58, 1901 (1985).

[3] D.L. Smith, C. Mailhiot, Rev. Modern Phys. 62, 173 (1990).

[4] B. Lai, G.M. Wells, F. Cerrina J. Vac. Sci. Technol. A 4, 977 (1986).

[5] V.S. Fomenko, Emission and Absorption Properties of Matters and Materials, Atomizdat, Moscow 1975 (in Russian).

[6] S.S. Borisova, I.F. Mikhailov, L.S. Palatnik, A.Yu. Sipatov, A.I. Fedorenko, L.P. Shpakovskaya, Kristallographia 34, 716 (1989) (in Russian).

[7] B.M. Clemens, J.G. Gay, Phys. Rev. B 35, 9337 (1987).

[8] F.F. Sizov, S.D. Darchuk, Phys. Status Solidi A 113, 107 (1989).

[9] K. Seeger, Semiconductor Physics, Springer-Verlag, Wien, New York 1973.

[10] G. Bastard, Phys. Rev. B 24, 5693 (1981). 